\title{
Chronic infection with Helicobacter pylori, Chlamydia pneumoniae, or cytomegalovirus: population based study of coronary heart disease
}

\author{
J Danesh, Y Wong, M Ward, J Muir
}

\begin{abstract}
Objective-To study possible associations between coronary heart disease and serological evidence of persistent infection with Helicobacter pylori, Chlamydia pneumoniae, or cytomegalovirus. Design-Population based, case-control study, nested within a randomised trial. Setting-Five general practices in Bedfordshire, UK.

Individuals-288 patients with incident or prevalent coronary heart disease and 704 age and sex matched controls.

Results-High concentrations of serum IgG antibodies to $H$ pylori were present in $54 \%$ of cases $v 46 \%$ of controls, with corresponding results for $C$ pneumoniae seropositivity (33\% $v 33 \%)$, and cytomegalovirus seropositivity (40\% $v$ 31\%). After adjustments for age, sex, smoking, indicators of socioeconomic status, and standard risk factors, the odds ratios $(95 \%$ confidence intervals) for coronary heart disease of seropositivity to these agents were: 1.28 (0.93 to 1.75$)$ for $H$ pylori, 0.95 (0.66 to 1.36 ) for $C$ pneumoniae, and 1.40 (0.96 to 2.05 ) for cytomegalovirus.

Conclusions-There is no good evidence of strong associations between coronary heart disease and serological markers of persistent infection with $\mathrm{H}$ pylori, $\mathrm{C}$ pneumoniae, or cytomegalovirus. To determine the existence of moderate associations between these agents and disease, however, larger scale studies will be needed that can keep residual confounders to a minimum.

(Heart 1999;81:245-247)
\end{abstract}

Keywords: ischaemic heart disease; Helicobacter pylori; Chlamydia pneumoniae; cytomegalovirus

It has been suggested that coronary heart disease may be associated with persistent bacterial or viral agents. ${ }^{1}$ Reports of such associations have raised the possibility that antiinfective treatments, such as a short course of antibiotics, might be able to prevent disease. ${ }^{23}$ Most studies have involved measurements for serum antibodies to Helicobacter pylori, Chlamydia pneumoniae, or cytomegalovirus, but only one report ${ }^{4}$ has measured antibodies to more than one of these agents in the same population, and none has reported measurement for all three. Also, previously reported metaanalyse ${ }^{5}{ }^{6}$ have indicated that published studies have generally been prone to biases and lacked adequate sample sizes. We have therefore conducted a population based, casecontrol study with measurements of markers for these three agents and for standard risk factors and possible confounding variables.

\section{Methods}

PARTICIPANTS AND LABORATORY METHODS

In the early 1990 s, 8100 volunteers aged 35 to 64 years at entry to a trial of nurse health checks in five Bedfordshire general practices completed questionnaires and gave non-fasting venous blood samples that were centrifuged and stored at $-80^{\circ} \mathrm{C}$ within 24 hours after venesection. ${ }^{7}$ Practice nurses took measurements of participants' height, weight, and blood pressure. For the present study, cases were defined on the basis of incident coronary heart disease death $(n=76)$ notified by national mortality statistics following entry to the trial (average follow up four years), or on the basis of self reported coronary heart disease at the baseline interview (103 cases with a history of myocardial infarction, and 109 with angina but no myocardial infarction) that could be verified by systematic searches of the medical notes by research nurses. Cases with more than one manifestation of coronary heart disease were classified by their most severe disease (that is, coronary heart disease death $>$ myocardial infarction $>$ angina). We randomly selected 704 people without a history of coronary heart disease from among the trial participants to match the cases on sex and age (within five years). Operators unaware of the case-control status of the blood samples measured serum lipid concentrations using standard assays, ${ }^{7}$ and IgG antibodies to $H$ pylori using a commercial kit (Orion; Pyloriset, Espoo, Finland), to C pneumoniae (whole organism antigen), and to cytomegalovirus (whole organism antigen) using time resolved fluorimetry (Delfia; Wallac, Turku, Finland). The coefficients of variation within and between assays for the infective agents were about $4 \%$ and $18 \%$, respectively. A validation study in 480 individuals from another study indicated good agreement with standard microimmunofluorescence for $C$ pneumoniae antibodies. ${ }^{8}$

\section{STATISTICS}

The distribution of $C$ pneumoniae titres was approximately normal, and cytomegalovirus titre distribution was approximately bimodal. As there are no generally agreed cut offs for seropositivity to $C$ pneumoniae or cytomegalovirus antibodies, analysis by thirds of titres was 
Table 1 Baseline characteristics of cases and controls

\begin{tabular}{|c|c|c|c|}
\hline Characteristic & $\begin{array}{l}\text { Cases } \\
(n=288)\end{array}$ & $\begin{array}{l}\text { Controls } \\
(n=704)\end{array}$ & $p$ Value \\
\hline \multicolumn{4}{|l|}{ Questionnaire } \\
\hline Age (years) & $59.2(5.9)$ & $59.0(6.1)$ & Matched \\
\hline Male & $200(69 \%)$ & $475(67 \%)$ & Matched \\
\hline Body mass index $\left(\mathrm{kg} / \mathrm{m}^{2}\right)$ & $27.4(4.4)$ & $26.1(3.8)$ & $<0.00001$ \\
\hline Current smokers & $155(54 \%)$ & $278(39 \%)$ & $<0.00001$ \\
\hline Treated diabetics & $23(8 \%)$ & $23(3 \%)$ & 0.001 \\
\hline Treated hypertensives & $133(46 \%)$ & $124(18 \%)$ & $<0.00001$ \\
\hline \multicolumn{4}{|l|}{ Blood sample } \\
\hline Total cholesterol $(\mathrm{mmol} / \mathrm{l})$ & $6.58(1.65)$ & $6.33(1.24)$ & 0.007 \\
\hline Low density lipoprotein cholesterol $(\mathrm{mmol} / \mathrm{l})$ & $3.69(1.75)$ & $3.67(1.48)$ & NS \\
\hline High density lipoprotein cholesterol ( $\mathrm{mmol} / \mathrm{l})$ & $1.18(0.44)$ & $1.30(0.42)$ & $<0.00001$ \\
\hline Triglycerides $(\mathrm{mmol} / \mathrm{l})$ & $2.48(1.40)$ & $2.15(1.33)$ & 0.0003 \\
\hline
\end{tabular}

Values are mean (SD) unless otherwise stated.

prespecified for each of these agents (that is, seropositive samples were defined as those in the top third of the control distribution, and seronegative samples as those in the bottom third), whereas the manufacturer's recommended cut off was used to determine $H$ pylori seropositivity. Associations of infective agents with coronary heart disease, risk factors, and other characteristics were investigated using $t$ tests, $\chi^{2}$ tests, and regression modelling (STATA Corporation, Texas, USA). The sample size was sufficient to detect odds ratios of 1.5 -fold or larger with $80 \%$ power at the 5\% level of significance, assuming about $50 \%$ seropositivity for each of the infective agents in the general population.

\section{Results}

As would be expected, there was a higher prevalence of known risk factors in cases with coronary heart disease than in controls (table $1)$.

Table 2 shows the prevalence of seropositivity of antibodies to each of the infective agents by case-control status. For $H$ pylori, measurements were available for 246 cases and 642 controls, of which 134 cases (54\%) and 294 controls $(46 \%)$ were seropositive. For $C$ pneumoniae, measurements were available for 288 cases and 704 controls, of which 94 cases $(33 \%)$ and 234 controls (33\%) had serum antibody titres in the top third of the distribution. For cytomegalovirus, measurements were available for 288 cases and 704 controls, of which 114 cases (40\%) and 221 controls $(31 \%)$ had serum antibody titres in the top third of the distribution. Adjustment for smoking and indicators of socioeconomic status moderately weakened each of these associa- tions (table 2), but adjustment for serum lipids, blood pressure, and other standard risk factors had little effect. None of the adjusted associations of coronary heart disease with serological evidence of these infections was highly significant. Varying the cut off for seropositivity around the prespecified levels for each agent did not change the estimates substantially. The data are too sparse to subdivide results reliably by possibly relevant characteristics, such as coronary heart disease type (for example, incident $v$ prevalent cases) or the presence of co-infections.

\section{Discussion}

More than 70 seroepidemiological studies have reported on associations between coronary heart disease and chronic infection with $H$ pylori, C pneumoniae, or cytomegalovirus. ${ }^{56}$ Most, however, have been based on small sample sizes, involved opportunistic control groups prone to selection biases, made few adjustments for possible confounders, and involved measurement for only one of these agents. It seems likely that chance effects, or the preferential publication of more extreme associations (that is, "publication bias"), or both, may at least partly account for the generally larger odds ratios reported in smaller studies without appropriate adjustment for confounders than in larger studies with population based controls. ${ }^{5610}$ It therefore remains uncertain whether infection with any of these agents is really associated with coronary heart disease. ${ }^{56}$

Our present population based, case-control study of 288 cases involved adjustment for standard vascular risk factors and several indicators of socioeconomic status. It confirmed the tendency to less extreme results in more reliable epidemiological studies of persistent infections and coronary heart disease, as we observed no strong association of coronary heart disease with levels of serum IgG antibodies to $H$ pylori, $C$ pneumoniae, or cytomegalovirus. For $H$ pylori, the adjusted odds ratio of 1.3 is compatible with the findings of a metaanalysis of four long term prospective reports involving a total 1441 cases that yielded a combined risk ratio of 1.2 for coronary heart disease death or myocardial infarction (95\% confidence interval 1.0 to 1.4$).^{6}{ }^{11-14}$ Evidence for a moderate association between $H$ pylori and coronary heart disease is, however, difficult to interpret even in population based studies, particularly as residual confounding by factors

Table 2 Prevalence of seropositivity for antibodies to some chronic infective agents

\begin{tabular}{|c|c|c|c|c|c|c|}
\hline \multirow[b]{2}{*}{ Agent } & \multicolumn{2}{|c|}{ Seropositive } & \multicolumn{2}{|c|}{ Seronegative ${ }^{\star}$} & \multicolumn{2}{|c|}{ Odds ratio and $95 \%$ confidence interval } \\
\hline & Cases & Controls & Cases & Controls & $\begin{array}{l}\text { Adjusted for age and } \\
\text { sex only }\end{array}$ & $\begin{array}{l}\text { Adjusted for age, sex, and } \\
\text { other factors } \dagger\end{array}$ \\
\hline H pylori & 134 & 294 & 112 & 348 & $1.41(1.05$ to 1.90$)$ & $1.28(0.93$ to 1.75$)$ \\
\hline C pneumoniae & 94 & 234 & 91 & 236 & $1.04(0.74$ to 1.48$)$ & $0.95(0.66$ to 1.36$)$ \\
\hline Cytomegalovirus & 114 & 221 & 82 & 245 & 1.57 (1.11 to 2.22$)$ & $1.40(0.96$ to 2.05$)$ \\
\hline
\end{tabular}

*For $C$ pneumoniae and cytomegalovirus antibodies, analysis by thirds of titres was prespecified-that is, seropositive samples were defined as those in the top third of the distribution, and seronegative samples as those in the bottom third, whereas manufacturer's recommended cut off was used to determine $H$ pylori sensitivity.

†Cigarette smoking (current, ex, never; number of cigarettes/day), markers of socioeconomic status (age at stopping education; occupation; housing tenure; car ownership; marital status; employment status), non-fasting serum total cholesterol, high density lipoprotein cholesterol, low density lipoprotein cholesterol, triglycerides, resting systolic and diastolic blood pressures, body mass index, and previous history of diabetes. 
related to socioeconomic status is likely. ${ }^{5}$ For $C$ pneumoniae, the three previously reported prospective seroepidemiological studies of coronary heart disease death or myocardial infarction (358 cases in all) collectively suggest a weakly positive association, ${ }^{615-17}$ albeit with very wide confidence limits, whereas the largest retrospective population based study of 302 cases did not report a positive association ${ }^{18}$ (and in fact it reported a non-significant inverse association). The estimate of the present study (odds ratio $1.0(0.7$ to 1.4$)$ ) is compatible with either no association or with a weakly positive association of $C$ pneumoniae seropositivity with coronary heart disease. With regard to cytomegalovirus, most of the previously reported studies have involved cases defined on the basis of vascular disease outside the coronary circulation or in transplanted hearts or coronary restenosis. ${ }^{5}{ }^{6}$ Our study doubles the available information from population based studies on cytomegalovirus and classic coronary heart disease, ${ }^{6}{ }^{19}$ and it does not suggest that there are strong associations.

The present study, together with the larger previously reported population based studies, suggests that the infective agents described in this report are unlikely to be strongly associated with coronary heart disease. It may still be important, however, to make a reliable assessment of possible moderate associations between these agents and coronary heart disease (for example, odds ratios $<1.5$ ), particularly if anti-infective interventions might be able to prevent some disease. Such studies would require substantially larger sample sizes than reported in available studies, as well as socially homogeneous populations to keep residual confounders to a minimum. Also, serial antibody measurements would help to correct for underestimation caused by fluctuations of serum antibody titres within individuals over time (especially for agents such as $C$ pneumoniae and cytomegalovirus, which are prone to reinfection and reactivation, respectively ${ }^{5}$ ). Results from such studies should be more informative than existing reports, particularly if they involve cases at younger ages where any real association between infections and coronary heart disease might be stronger than at older ages. ${ }^{5}$
Alice Fuller helped with data management, and Jenny Jay and Janet Robertson audited clinical notes and retrieved blood samples. JD is supported by a Merton College Junior Research Fellowship and a Frohlich award; YW and MW are supported by the British Heart Foundation and the Wessex Heartbeat Trust.

1 Libby P, Egan D, Skarlatos S. Roles of infectious agents in atherosclerosis and restenosis: an assessment of the vidence and need for future research. Circulation 1997;96:4095-103.

2 Gupta S, Leatham EW, Carrington D, et al. Elevated Chlamydia pneumoniae antibodies, cardiovascular events, and azithromycin in male survivors of myocardial infarction. Circulation 1997;96:404-7.

3 Gurfinkel E, Bozovich G, Daroca A, et al. Randomised trial of roxithromycin in non-Q-wave coronary syndromes: ROXIS pilot study. Lancet 1997;350:404-7.

4 Patel P, Mendall MA, Carrington D, et al. Association of Helicobacter pylori and Chlamydia pneumoniae infections with coronary heart disease and cardiovascular risk factors. BMF 1995;311:711-14.

5 Danesh J, Collins R, Peto R. Chronic infections and coronary heart disease: is there a link? Lancet 1997;350:430-6.

6 Danesh J, Appleby P. Persistent infection and vascular disease: a systematic review. Expert Opin Invest Drugs 1998; 7:691-713.

7 Imperial Cancer Research Fund OXCHECK Study Group. Prevalence of risk factors for heart disease in OXCHECK trial: implication for screening in primary care. BMF 1991; 302:1057-60.

8 Wong YK, Ward M. The species specificity of the microimmunofluorescence antibody test and comparisons with time resolved fluoroscopic immunoassay. F Clin Pathol. [In press].

9 Easterbrook P, Berlin J, Gopalan R, et al. Publication bias in clinical research. Lancet 1991;337:867-72.

0 Danesh J, Peto R. Risk factors for coronary heart disease and infection with Helicobacter pylori: meta-analysis of 18 studies. BMF 1998;316:1130-2.

11 Wald NJ, Law MR, Morris JK, et al. Helicobacter pylori infection and mortality from ischaemic heart disease: negative results from a large, prospective study. BMF 1997;315: 1199-201.

12 Aromaa A, Knekt P, Reunanen A, et al. Helicobacter pylori and the risk of myocardial infarction [abstract]. Gut 1996; 39(suppl 2):A91.

13 Whincup PH, Mendall MA, Perry IJ, et al. Prospective relations between Helicobacter pylori infection, coronary heart disease, and stroke in middle-aged men. Heart 1996;75: 568-72.

14 Folsom AR, Nieto FJ, Sorlie P, et al. No association of Helicobacter pylori seropositivity with coronary heart disease in a prospective study [abstract]. Circulation 1997;96(suppl I):I-100.

15 Miettinen H, Lehto S, Saikku P, et al. Association of Chlamydia pneumoniae and acute coronary heart disease events in non-insulin dependent diabetic and non-diabetic events in non-insulin dependent diabetic and no

16 Saikku P, Leinonen M, Tenkanen L, et al. Chronic Chlamydia pneumoniae infection as a risk factor for coronary heart disease in the Helsinki heart study. Ann Intern Med 1992;116:273-8.

17 Ossewarde JM, Vallinga C, Feskens EJM, et al. Infection with Chlamydia pneumoniae as a risk factor for coronary heart disease (the Zutphen elderly study) [abstract]. Atherosclerosis 1995;115(suppl):S13.

18 Kark JD, Leionen M, Paltiel O. Chlamydia pneumoniae and acute myocardial infarction in Jerusalem. Int $\mathcal{F}$ Epidemiol 1997;4:730-8.

19 Viik-Kajander M, Roivainen M, Manninen V, et al. Herpes virus infections and the risk of myocardial infarction and coronary death [abstract]. Circulation 1997;96(suppl I):I374. 